\title{
ARTICLE Neural predictors of treatment response to brain stimulation and psychological therapy in depression: a double-blind randomized controlled trial
}

\author{
Camilla L. Nord iD $^{1,2}$, D. Chamith Halahakoon ${ }^{1,3}$, Tarun Limbachya ${ }^{4}$, Caroline Charpentier ${ }^{1,5}$, Níall Lally ${ }^{1,6,7}$, Vincent Walsh ${ }^{1}$, \\ Judy Leibowitz ${ }^{4}$, Stephen Pilling ${ }^{4,8}$ and Jonathan P. Roiser (D)
}

Standard depression treatments, including antidepressant medication and cognitive behavioural therapy (CBT), are ineffective for many patients. Prefrontal transcranial direct current stimulation (tDCS) has been proposed as an alternative treatment, but has shown inconsistent efficacy for depression, and its mechanisms are poorly understood. We recruited unmedicated patients with major depressive disorder ( $N=71$ approached; $N=39$ randomised) for a mechanistic, double-blind, randomized controlled trial consisting of eight weekly sessions of prefrontal tDCS administered to the left prefrontal cortex prior to CBT. We probed (1) whether tDCS improved the efficacy of CBT relative to sham stimulation; and (2) whether neural measures predicted clinical response. We found a modest and non-significant effect of tDCS on clinical outcome over and above CBT (active: 50\%; sham: $31.6 \%$; odds ratio: $2.16,95 \% \mathrm{Cl}=0.59-7.99$ ), but a strong relationship, predicted a priori, between baseline activation during a working memory task in the stimulated prefrontal region and symptom improvement. Repeating our analyses of symptom outcome splitting the sample according to this biomarker revealed that tDCS was significantly superior to sham in individuals with high left prefrontal cortex activation at baseline; we also show $86 \%$ accuracy in predicting clinical response using this measure. Exploratory analyses revealed several other regions where activation at baseline was associated with subsequent response to CBT, irrespective of tDCS. This mechanistic trial revealed variable, but predictable, clinical effects of prefrontal tDCS combined with CBT for depression. We have discovered a potential explanation for this variability: individual differences in baseline activation of the region stimulated. Such a biomarker could potentially be used to pre-select patients for trials and, eventually, in the clinic.

Neuropsychopharmacology (2019) 44:1613-1622; https://doi.org/10.1038/s41386-019-0401-0

\section{INTRODUCTION}

Cognitive behavioural therapy (CBT) is a common, effective treatment for depression [1]. Nevertheless, only $60 \%$ of patients show an adequate response to therapy; even fewer achieve remission [2]. Some researchers have suggested improving CBT response with augmentative strategies, such as cognitiveenhancing drugs [3]. More recently, non-invasive brain stimulation, including repetitive transcranial magnetic stimulation (rTMS) [4] and transcranial direct current stimulation (tDCS) [5], has been suggested as a strategy to enhance response to $\mathrm{CBT}$.

Both rTMS and tDCS have been applied as treatments for depression over the dorsolateral prefrontal cortex (DLPFC), a region disrupted in both currently depressed [6] and remitted patients $[7,8]$. Stimulation of the DLPFC directly affects its activation, as well that of distal regions including the cingulate cortex $[9,10]$. Previous trials have shown that prefrontal tDCS has a moderately strong antidepressant effect [11-16].
Recently, a large multi-centre trial failed to show any evidence in support of tDCS, even finding a potentially detrimental effect for unipolar depression [17]. This inconsistency echoes non-clinical experiments. Initially, many results suggested that prefrontal tDCS enhances cognitive performance, including planning [18], insight [19], and cognitive control during and after tDCS, in both healthy $[20,21]$ and depressed [22] individuals. However, the cognitive effects of tDCS also appear highly variable [21, 23, 24]; a quantitative review suggested there were no consistent effect of tDCS on any cognitive process in healthy individuals [23] (for a critique of this review, see [25]).

If prefrontal tDCS improves cognitive control, it may improve patients' ability to benefit from CBT, which itself entails challenging cognitive processing, including planning and working memory [1]. This is supported by preliminary evidence that tDCS enhances the antidepressant effects of cognitive control training [26] and, in a small feasibility trial $(N=10)$, internet-based CBT [27]. However, given the inconsistent evidence for tDCS in

\footnotetext{
${ }^{1}$ Institute of Cognitive Neuroscience, University College London, London, UK; ${ }^{2}$ MRC Cognition and Brain Sciences Unit, University of Cambridge, Cambridge, UK; ${ }^{3}$ Department of Psychiatry, University of Oxford, Oxford, UK; ${ }^{4}$ Camden and Islington NHS Foundation Trust, London, UK; ${ }^{5}$ Division of the Humanities and Social Sciences, California Institute of Technology, California, USA; ${ }^{6}$ Warwick Medical School, University of Warwick, Coventry, UK; ${ }^{7}$ Experimental Therapeutics \& Pathophysiology Branch, Intramural Research Program,

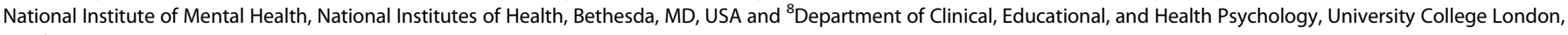
London, UK

Correspondence: Camilla L. Nord (Camilla.Nord@mrc-cbu.cam.ac.uk)

Trial Registration: The trial was registered at clinicatrials.gov, registration number NCT01875419; URL: https:/clinicaltrials.gov/ct2/show/NCT01875419?term=NCT01875419\&rank=1
}

Received: 16 January 2019 Revised: 21 March 2019 Accepted: 15 April 2019

Published online: 30 April 2019 


\section{4}

both cognition [23] and depression [17], it is also likely there are substantial individual differences in response to tDCSenhanced CBT.

A key priority for psychiatric research is identifying objective measures to help optimize treatment selection [28]. There is preliminary evidence that pre-treatment neural activation (measured with functional magnetic resonance imaging: $\mathrm{fMRI}$ ) can predict response to antidepressant medication and CBT [29]. However, studies investigating such associations invariably lack comparison groups and randomization to groups. To identify biomarkers unambiguously, mechanistic measurements need to be incorporated into randomized controlled trials (RCTs).

A strong candidate biomarker for response to non-invasive brain stimulation in depression is activation of the region stimulated (usually the DLPFC): experimentalists have hypothesized that baseline DLPFC activity might mediate the cognitive effects of prefrontal tDCS on cognition [24], and one small study in depressed patients reported that higher pre-treatment DLPFC resting-state perfusion was associated with better clinical response to DLPFC rTMS [30].

Therefore we conducted a mechanistic, proof-of-principle RCT of prefrontal tDCS to augment CBT in unmedicated patients with unipolar depression. We hoped to capitalise on the sustained ( 90 min) aftereffects of tDCS on cortical excitability by delivering stimulation immediately prior to weekly CBT sessions [31]. Our study had two key aims: (1) to assess whether tDCS augments the ability of CBT to treat depression over and above sham (placebo) stimulation; and (2) to explore putative pre-treatment biomarkers. We also aimed to identify the cognitive and neural changes resulting from combined CBT and tDCS. We hypothesized that (1) tDCS would improve response to CBT; and (2) that higher pretreatment activation in the DLPFC would predict better response to combined CBT and tDCS.

\section{MATERIALS AND METHODS}

\section{Participants}

We randomized 39 unmedicated patients with major depressive disorder (Hamilton Rating Scale for Depression (HAM-D) > 17) to receive real $(N=20)$ or sham $(N=19)$ tDCS. Patients were recruited through the Camden and Islington NHS Foundation Trust Improving Access to Psychological Treatment (IAPT) service within the UK National Health Service (NHS). Patients, investigators, and therapists were blind to tDCS condition for the duration of the trial. Six patients did not complete the intervention (defined as $\geq 7 / 8$ sessions: 1 active; 5 sham); dropout rates differed marginally (non-significantly) between groups $(p=0.091)$; to account for potential non-random dropout we employed intention-to-treat analyses as our primary analysis. Thirty-one patients received eight sessions of CBT and two patients received seven sessions of CBT. See Fig. 1 for CONSORT diagram and procedure.

Only patients meeting criteria for at least moderate depression where the IAPT service assigned one-on-one cognitive behavioural therapy (CBT) for depression were included in the trial (see Supplemental Materials for full participant criteria). The study was approved by the London Queen Square NHS Research Ethics Committee (ID: 13/LO/1028); subjects' consent was obtained in accordance with the Declaration of Helsinki.

\section{Protocol}

The study protocol was registered on clinicaltrials.gov (NCT01875419). See Fig. 1a for CONSORT diagram. See Fig. 1b for trial protocol, consisting of a screening session, fMRI scans (before and after intervention), and randomization (using custom-written MATLAB code by a researcher not involved in the trial). See Supplemental Materials for the full protocol.
Imaging details

The MRI protocol included a T1-weighted anatomical scan and two T2*-weighted functional scans ( $\mathrm{n}$-back working memory task [21], practiced at the screening session to ensure participants could perform the task; and an emotion processing task [32], where participants discriminated the gender of fearful, happy, and neutral faces). See Supplemental Materials for full details.

\section{Stimulation procedure}

Immediately preceding each CBT session, a $1 \mathrm{~mA}$ constant current fronto-extracephalic montage was delivered to the left prefrontal cortex (anode on F3 on the international 10-20 electroencephalography (EEG) system using an EEG cap for placement), secured in place with an elasticated head-strap; cathode on ipsilateral deltoid). Although this delivers relatively diffuse prefrontal stimulation [33], an open-label, preliminary study comparing bifrontal, and fronto-extracephalic tDCS in depression found that fronto-extracephalic tDCS was more effective [34] motivating our choice (note, however, that this study may have been underpowered: $N=11$ ). We were restricted to $1 \mathrm{~mA}$ by our ethics committee; however, a recent study found that only unilateral 1 $\mathrm{mA}$ stimulation (and not bilateral $1 \mathrm{~mA}$ or $2 \mathrm{~mA}$ ) caused sustained increases in corticospinal excitability [35].

The stimulation, delivered using a neuroConn DC-stimulator, lasted for $20 \mathrm{~min}$. Sham stimulation involved $30 \mathrm{~s}$ of direct current followed by $1170 \mathrm{~s}$ without, which produces a similar sensation to active stimulation (most sensations are felt in the first $30 \mathrm{~s}$ during ramping up), resulting in effective blinding at $1 \mathrm{~mA}$ [36]. Both electrodes were $35 \mathrm{~cm}^{2}(7 \times 5 \mathrm{~cm})$, rubber, and placed inside electrode sponges that had been briefly soaked in saline to conduct the current. The polarity of the device was independently verified using a resistor and volt-meter. For practicality, tDCS was administered prior to CBT. Following stimulation, patients completed the Beck Depression Inventory (BDI), Beck Anxiety Inventory (BAl), and Snaith-Hamilton Pleasure Scale (SHAPS).

Patients performed the $n$-back working memory task during stimulation (in real and sham stimulation conditions), as previous studies reported enhanced cognitive effects of tDCS when delivered concurrently with a working memory paradigm [26]. The $n$-back task was identical to the version patients were previously trained on and had performed inside the scanner.

\section{Therapy procedure}

Immediately following each tDCS session, patients received a 1-h CBT intervention for depression, as described previously [37]. Sessions occurred a minimum of 6 days apart.

We decreased variability in CBT delivery by working with a selected team of therapists, doctoral-level clinical psychologists working in the NHS IAPT service, which itself has standardised training and CBT delivery. To ensure adherence to protocol, therapists also received regular supervision of CBT sessions by supervisors accredited by the British Association of Behavioural and Cognitive Psychotherapists (BABCP). To increase consistency of $\mathrm{CBT}$, we ran a day-long expert-led training for all therapists before recruitment.

\section{Side effects and blinding}

At each session, following tDCS, patients filled out the tDCS Adverse Events Questionnaire [38], and guessed their stimulation condition. At four sessions, the Adverse Events questionnaire was not completed due to time constraints.

\section{Analyses}

Our primary clinical outcome measure was response rate according to the HAM-D, defined as a reduction of at least $50 \%$ from baseline; we also report remission rate, defined as a HAM-D score of 7 or below. We analysed our primary outcome with an intent-to-treat analysis (accounting for missing values at follow-up 
A

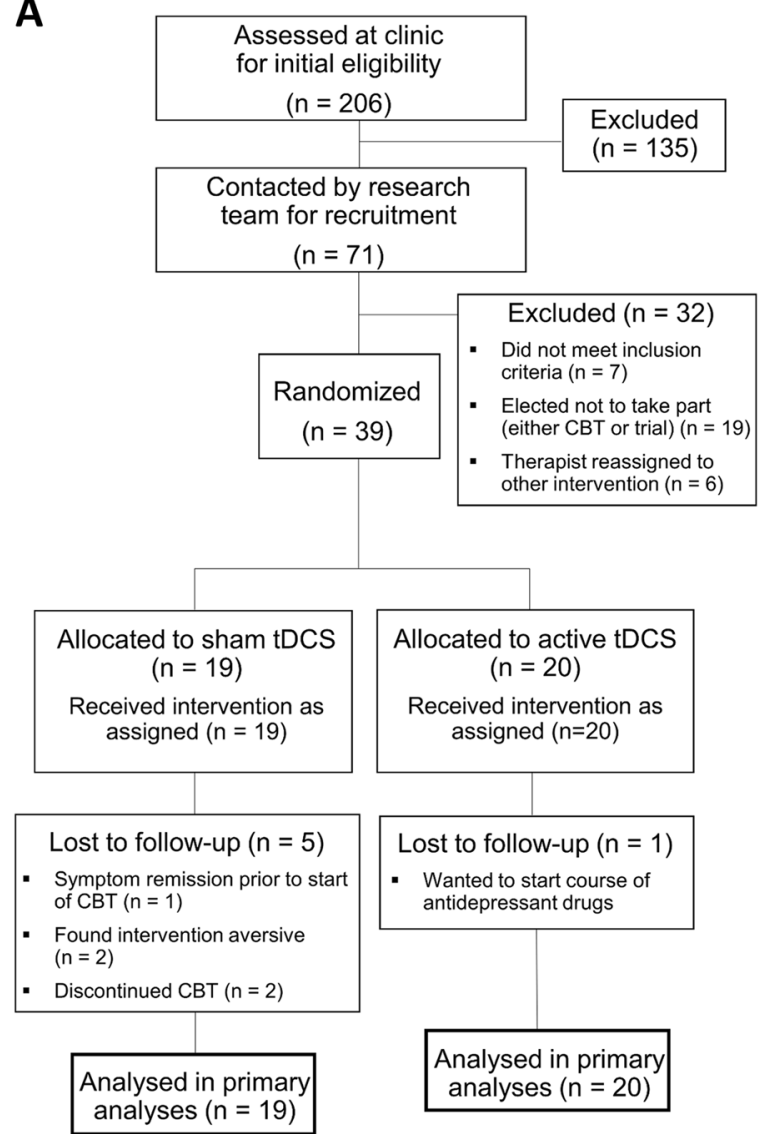

B

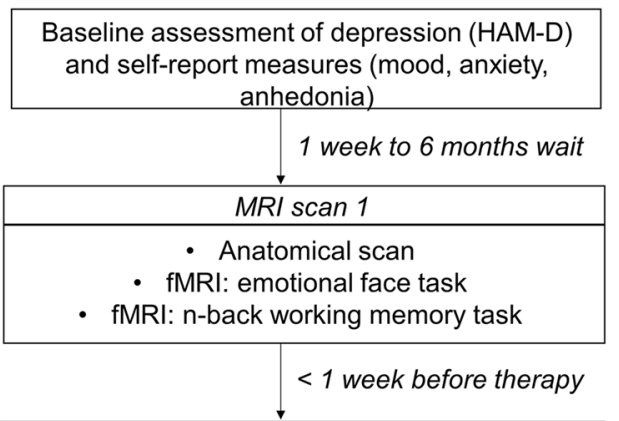

Patients randomized to receive real or sham $\operatorname{tDCS}(n=39)$

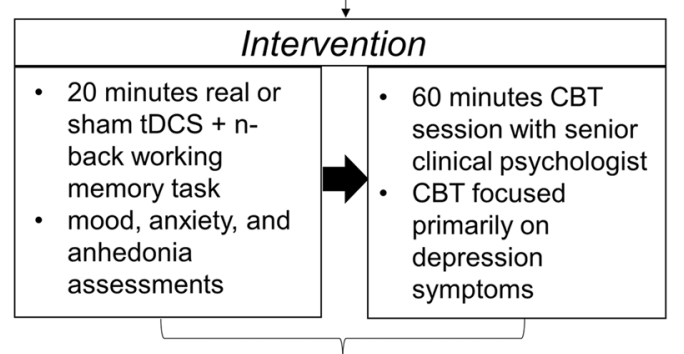

Continued for sessions (at least 1 week apart), until completion of trial or dropout

(8 sessions: $n=31 ; 7$ sessions: $n=2$; dropout: $n=6$ )

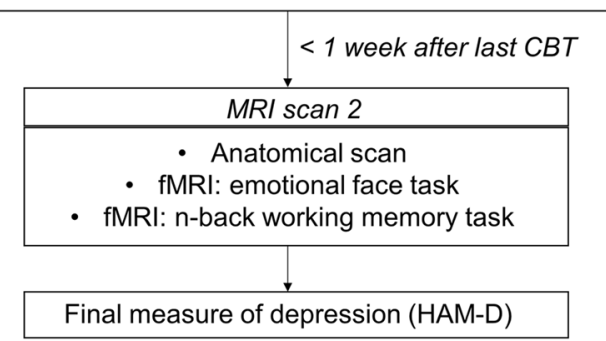

Fig. 1 CONSORT diagram describing recruitment, randomization, and attrition in the clinical trial (a) and depiction of trial procedure (b). CBT cognitive behavioural therapy, tDCS transcranial direct current stimulation, HAM-D Hamilton Rating Scale for Depression, fMRI functional magnetic resonance imaging

using last observation carried forward: $N=39$ ) as we could not assume attrition occurred completely at random. We analysed secondary outcome measures (BDI, BAI, SHAPS, and working memory performance $d^{\prime}$ (previously described [21])) with linear mixed models, which accounts for missing data by estimating the trajectory of change for the subjects who provided data (see Supplemental Materials for details).

For all measures, we repeated this analysis using the sample of patients who completed the treatment.

fMRI analyses (on the sample who completed the trial) were performed in SPM12 (https://www.fil.ion.ucl.ac.uk/spm/). Our primary analyses used anatomically-defined regions of interest (ROIs): for the emotion processing task, the left and right amygdala, subgenual anterior cingulate cortex, and L-DLPFC (contrasts: fearful $>$ neutral faces and happy $>$ neutral faces); for the n-back task, R-and L-DLPFC (constrast: 3-back>1-back). L-DLPFC was identified by placing a $10 \mathrm{~mm}$ sphere on coordinates from a meta-analysis of working memory tasks, the majority of which used the n-back $(-44,20,30)$ [6]; R-DLPFC was defined using the corresponding contralateral coordinate $(44,20,30)$. We additionally conducted an exploratory whole-brain flexible factorial analysis (with an initial cluster-forming threshold of $p<0.001$, uncorrected). For these exploratory analyses, we used a Bonferroni-corrected significance threshold of $p=0.025$ (FWEcorrected), reporting significance at the cluster- or voxel-level.

Outcome prediction analysis

We first mean-corrected all predictor variables: R- and L-DLPFC activation; R- and L-amygdala activation; sgACC activation; $\mathrm{n}$-back $d^{\prime}$; SHAPS; BAl; and HAM-D. Note all predictor variables were acquired prior to randomisation.

Our primary analyses tested whether pre-randomization activation averaged within each ROI (the L-DLPFC for the n-back task; the amygdalae and sgACC for the emotional faces task; all analysed in separate regressions) could predict the degree of clinical improvement (HAM-D percent change). Each regression also included the fixed effect of stimulation condition and the interaction between stimulation condition and baseline ROI activation. If this model was significant, we then conducted sensitivity analyses that tested whether the prediction remained significant following inclusion of additional variables in the model (HAM-D, BAI, and SHAPS scores, as well as n-back performance, and their interactions with stimulation group) (see Supplemental Materials for details). 
1616

Power calculation

This was a mechanistic, proof-of-principle trial powered to detect a large effect size, as reported in a previous trial of tDCS in antidepressant-free patients (Hedges' $g=0.988$ ) [11] and report of tDCS enhancing n-back performance (Cohen's $d=1.42$ ) [21]. We had $80 \%$ power to detect $w=0.45\left(X^{2}\right.$ test; alpha $\left.=0.05\right)$ for our primary clinical outcome variable (HAM-D), which was binary. For continuous variables, including our mechanistic (fMRI) measures, we had $80 \%$ power to detect $d=1.0$ (independent samples $t$-test; alpha $=0.05$ (two-tailed)).

\section{RESULTS}

Clinical and demographic data are presented in Table 1. There were no differences in baseline generalised anxiety disorder (GAD) between patients subsequently assigned to real and sham conditions: $14 / 20$ patients assigned to real stimulation, and 13/19 patients assigned to sham stimulation, met criteria for $\operatorname{GAD}\left(X^{2}=0.011, p=0.915\right)$.

Assessment of blinding and side effects

$38.5 \%$ of patients correctly guessed the stimulation condition, with no significant difference in the proportion of active and sham guesses between groups $\left(X^{2}=1.64, p=0.301\right)$, suggesting that blinding was effective. See Supplemental Materials details and full side effects.

Primary clinical outcome

Using an intention-to-treat analysis (last observation carried forward), a greater proportion of patients responded (active: 50\%; sham: $31.6 \%$; odds ratio: $2.16,95 \% \mathrm{Cl}=0.59-7.99)$ and remitted (active: $30 \%$; sham: $10.5 \%$; odds ratio: $3.65,95 \% \mathrm{Cl}=$ 0.63-20.96) following CBT with active than with sham tDCS

Table 1. Participant characteristics by stimulation condition

\begin{tabular}{lll}
\hline & Active tDCS & Sham tDCS \\
\hline $\mathrm{N}$ & 20 & 19 \\
$\%$ female & 45.00 & 57.89 \\
Age & $35.60(12.91)$ & $31.05(8.17)$ \\
FSIQ & $110.10(7.49)$ & $105.15(7.54)$ \\
Baseline HAM-D & $21.95(3.20)$ & $21.05(3.27)$ \\
Baseline BDI & $25.70(8.01)$ & $27.79(5.34)$ \\
Baseline SHAPS & $19.85(7.25)$ & $18.05(7.25)$ \\
Baseline BAI & $25.70(13.57)$ & $25.47(12.06)$ \\
Age of onset & $22.80(10.09)$ & $18.37(8.23)$ \\
No. episodes & $2.50(1.67)$ & $3.05(1.58)$ \\
$\%$ hospitalized & 15.00 & 15.79 \\
$\%$ suicide attempt & 30.00 & 21.05 \\
$\%$ past ADM & 55.00 & 42.10 \\
$\%$ past PT & 50.00 & 78.90 \\
$\%$ baseline GAD & 75 & 68 \\
\hline
\end{tabular}

Other than $\mathrm{N}$ and percentage data, figures represent means (standard deviations)

FSIQ Full Scale Intelligence Quotient (calculated from Weschler Test of Adult Reading), HAM-D Hamilton Rating Scale for Depression, BDI Beck Depression Inventory, BAl Beck Anxiety Inventory, SHAPS Snaith-Hamilton Pleasure Scale, No. number, \% past ADM percent of patients with previous history of antidepressant medication use (no patients were currentlymedicated), \% past PT percent of patients with a previous history of psychological therapy (all patients were engaged in CBT during the trial), \% baseline GAD percent of patients who met criteria for generalised anxiety disorder (GAD) at baseline (defined as a $50 \%$ or greater reduction on the Hamilton Rating Scale for Depression, HAM-D, and a HAM-D of less than 7, respectively). However, these differences did not achieve statistical significance (response: $X^{2}=1.37, p=0.24, \varphi=0.187$; remission: $X^{2}=2.27, p=0.13, \varphi=0.241$ (see Fig. 2a). We repeated these analyses for the sample of patients who completed the trial (response: active: $52.6 \%$; sham: $42.9 \%$; odds ratio: $1.48 ; X^{2}=0.308$, $p=0.579, \varphi=0.184$; remission: active: $31.6 \%$; sham: $14.3 \%$; odds ratio: $\left.2.76 ; X^{2}=1.313, p=0.252, \varphi=0.090\right)$.

Secondary clinical outcomes

HAM-D scores reduced significantly from pre- to post-intervention $\left(F(1,37)=56.09, \quad p<0.001, \eta_{p}{ }^{2}=0.603\right.$ ) (mean difference 9.30 $(S D=6.82)$ in active, $6.68(S D=6.49)$ in sham), but the main effect of stimulation condition $(F(1,37)=0.112, p=0.739)$ and the stimulation-by-time interaction $(F(1,37)=1.50, p=0.228)$ were non-significant (Fig. 2b). The results were similar for completers $(N=33)$. Including therapist as a covariate in this analysis produced similar results (see Supplemental Materials). There were no significant effects of stimulation condition on questionnaire measures or working memory performance (all $p>0.1$ ) (see Supplemental Materials for full results, and in-scanner task performance).

Neural activation: n-back working memory task

$R O I$ analysis (a priori). Bilateral DLPFC activation increased after the intervention (main effect of time: $F(1,31)=20.95, p<0.001$, $\left.\eta_{\mathrm{p}}{ }^{2}=0.403\right)$, but the interaction with group was non-significant $(F(1,31)=0.363, p=0.551)$ (Fig. 2). At baseline patients showed significantly lower bilateral DLPFC activation than 30 matched healthy controls $\left(F(1,61)=8.24, p=0.006, \eta_{p}{ }^{2}=0.119\right)$, but there was no significant difference from healthy controls post-intervention $(F(1,61)=0.191, p=0.664)$. There was no association between increased bilateral DLPFC activation and $\mathrm{n}$-back improvement $(\mathrm{r}(33)=0.064, p=0.725)$. Full results in Supplemental Materials.

Whole-brain analysis (exploratory). Activation increased significantly from pre- to post-intervention in bilateral parietal cortex and bilateral DLPFC, and decreased significantly in medial prefrontal cortex, extending into perigenual ACC (all $p<0.001$, cluster-level FWE-corrected; Fig. 2e and Supplemental Table 2). Patients receiving active stimulation also had significantly greater activation in right posterior parietal cortex (rPPC) compared to sham ( $p=0.02$ voxel-level FWE-corrected: Fig. 2f). rPPC activation increased over time in the active condition $(t(18)=2.96, p=0.008)$ but decreased numerically in the sham condition $(t(13)=1.98$, $p=0.069$ ) (group-by-time interaction: $F(1,31)=11.74, p=0.002$; Fig. 2f). Note that since the contrast used to identify this result was the main effect of group, the group-by-time interaction is orthogonal, meaning this is not a circular analysis.

Neural activation: emotion processing task

Amygdala $\mathrm{ROI}$ analysis (a priori). We found a significant time-bysimulation condition interaction in the amygdala $(F(1,31)=5.04$, $\left.p=0.032, \eta_{p}{ }^{2}=0.140\right)$ : those in the sham group showed decreased amygdala activation (averaged across contrasts) at post- relative to pre-intervention $(t(14)=2.63, p=0.021)$, while those in the active group did not $(t(19)=0.605, p=0.553)$. Independent samples $t$-tests revealed marginal (non-significant) differences between the active and sham conditions pre- $(t(31)=$ 1.74, $p=0.092)$ and post-intervention $(t(31)=1.96, p=0.059)$, such that there was numerically lower baseline amygdala activation and numerically higher post-intervention amygdala activation in patients subsequently assigned to the active condition (compared to those assigned to sham). No other main or interaction effects reached significance (see Fig. 3; full results described in Supplemental Materials). 
L-DLPFC ROI analysis (a priori). Similarly, there was a significant interaction between time and stimulation condition $(F(1,31)=$ $\left.10.95, p=0.002, \eta_{p}^{2}=0.261\right)$. In patients receiving active stimulation, L-DLPFC activation (collapsing across fear and happy contrasts) marginally (non-significantly) increased from pre-to post-intervention $(t(18)=2.06, p=0.055)$; in patients receiving sham stimulation, left DLPFC activation decreased significantly $(t(13)=2.62, p=0.021)$. Independent samples $t$-tests revealed a marginal (non-significant) difference between the stimulation conditions at baseline $(t(31)=1.88, p=0.07)$, but postintervention there was significantly greater L-DLPFC activation in the active compared to the sham stimulation group $(t(31)=2.08$,
A

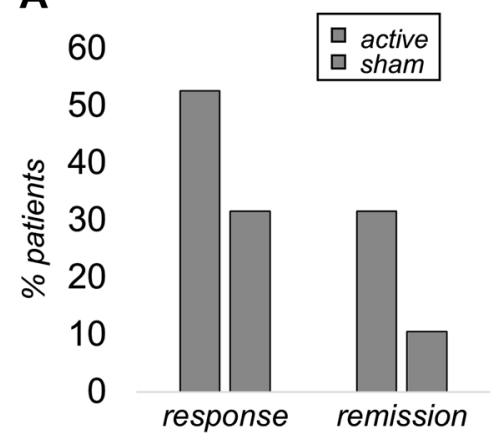

C

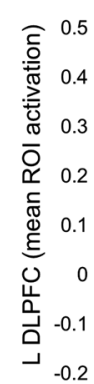

B

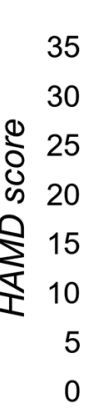

D

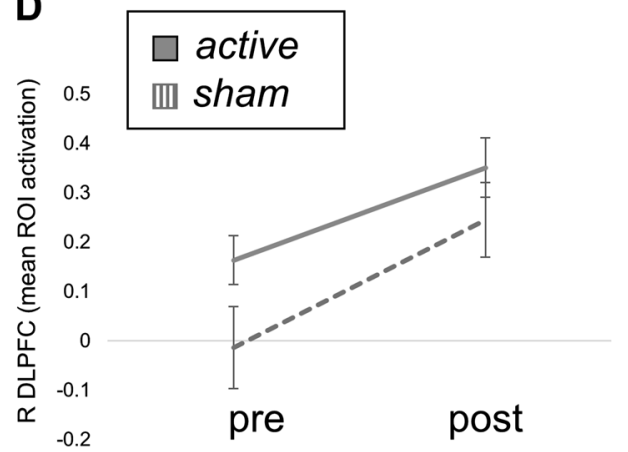

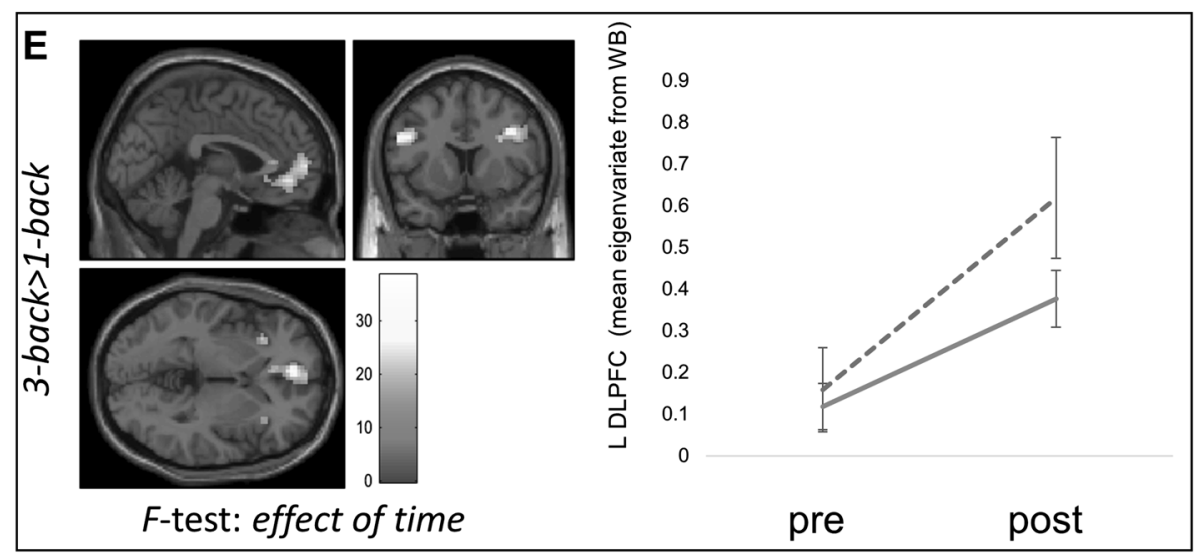

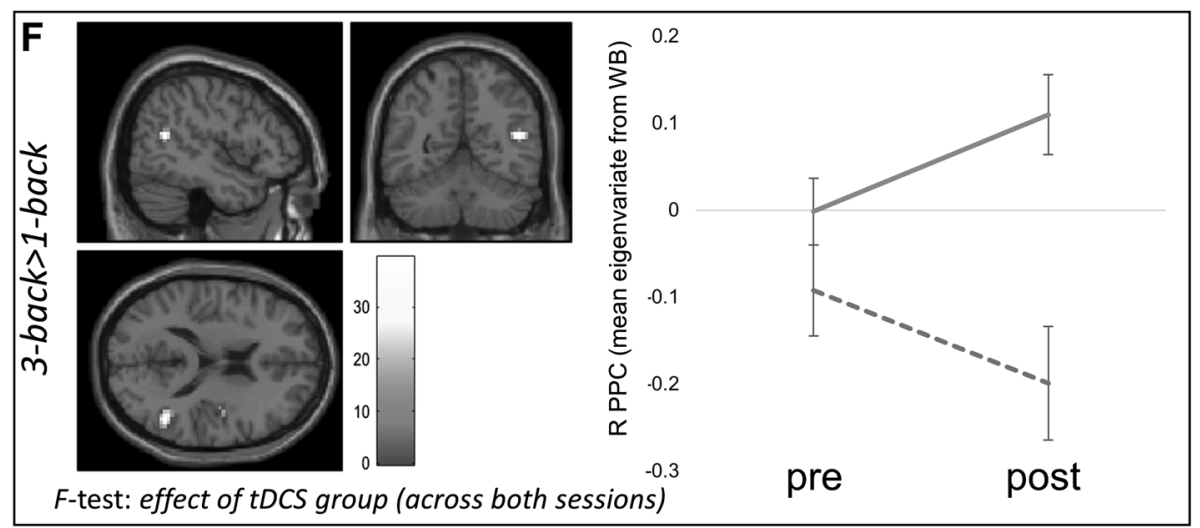


Fig. 2 Effect of intervention on depressive symptoms and brain activation. a Proportion of patients responding and remitting following CBT with active and sham tDCS. These differences did not achieve statistical significance. b HAM-D scores reduced significantly from pre- to postintervention, but the main effect of stimulation condition and the interaction were non-significant. C\&D: Effect of intervention on neural activation during working memory (contrast: 3-back > 1-back). Activation in left (c) and right (d) dorsolateral prefrontal cortex (DLPFC) a priori regions of interest pre- and post-intervention (orange solid lines = active tDCS; green dotted lines $=$ sham tDCS). e Whole-brain (WB) analysis of the effect of time (pre- versus post-intervention) and plotted eigenvariate of this main effect. e, left panel: we found significant changes in activation over time in bilateral DLPFC (increased over time: coronal section; $p<0.001$ (uncorrected) cluster-forming threshold, WB clusterlevel FWE-corrected) and medial PFC/perigenual anterior cingulate cortex (decreased over time: sagittal and axial sections; $p<0.001$ (uncorrected) cluster-forming threshold, WB cluster-level FWE-corrected). e, right panel: for illustrative purposes, eigenvariate values extracted from the L-DLPFC cluster displayed in the left panel are plotted. $f$ WB effect of group (greater in active than sham tDCS condition) in the right posterior parietal cortex (rPPC) ( $p=0.02$ WB voxel-level FWE corrected: $\mathbf{f}$, left), and plotted eigenvariate of this rPPC cluster (f, right). Overlays are displayed at $p<0.001$ (uncorrected) and colour bars indicate F-values. Error bars indicate standard errors of the mean

$p=0.046)$. No other main or interaction effects reached significance (see Supplemental Materials).

\section{sgACC ROI analysis (a priori). There were no significant effects}

Whole-brain analysis (exploratory). There were no significant main or interaction effects of stimulation condition on emotion processing at our corrected threshold of $p=0.025$ (clusteror voxel-level) (see Supplemental Materials and Supplemental Table 3).

Prediction of outcome

ROI analysis (a priori). In the L-DLPFC model (n-back: 3-back > 1back contrast), pre-treatment activation was significantly positively associated with subsequent percentage improvement in HAM-D score $\left(F(1,29)=12.77, p=0.001, \eta_{p}{ }^{2}=0.306\right)$. There was also a significant interaction between activation and stimulation condition $\left(F(1,29)=6.83, p=0.014, \eta_{p}^{2}=0.191\right)$. This remained significant after controlling for baseline symptom and working memory scores (main effect: $F(1,26)=29.90, p<0.001, \eta_{p}{ }^{2}=$ $0.535)$; interaction: $\left(F(1,26)=10.87, p=0.003, \eta_{p}{ }^{2}=0.295\right)$ ) (see Supplemental Materials).

Pre-treatment activation in L-DLPFC was significantly positively associated with subsequent symptomatic improvement in the active $(r=0.711, p=0.001)$ but not sham $(r=0.205, p=$ 0.482 ) condition (Fig. 4a). Note, $n$-back performance did not predict percentage improvement $(r=0.112, p=0.536)$. Baseline L-DLPFC activation was not associated with baseline HAM-D severity $(p>0.1)$.

In the right amygdala model (emotion processing: fearful > neutral contrast), pre-treatment activation was significantly positively associated with subsequent percentage improvement in HAM-D score across both groups $(F(1,29)=7.69, p=0.010$, $\left.\eta_{\mathrm{p}}{ }^{2}=0.210\right)$; the interaction with stimulation group was nonsignificant $(F(1,29)=0.54, p=0.469)$ (Fig. 4b).

No other regions or their interaction with stimulation condition were significantly associated with clinical outcome (all $p>0.1$ ); therefore, we did not proceed with sensitivity or reliability analyses.

Whole-brain analysis (exploratory). Higher pre-treatment activation in the left posterior parietal cortex during working memory (L PPC, $p=0.027$, FWE cluster-corrected) and higher left rostral PFC during emotion processing (fearful > neutral contrast; $p=$ 0.001 , FWE cluster-corrected) were positively associated with greater subsequent percentage improvement in HAM-D across both groups (Fig. 4c-f); however, only the latter survived the Bonferroni-corrected threshold of $p=0.025$ FWE. No interactions with stimulation group survived whole-brain correction for multiple comparisons (see Supplemental Tables 4-6).

Post-hoc area-under-curve (AUC) analysis: L-DLPFC prediction of clinical response

We repeated our primary analyses after dividing the sample into low and high-L-DLPFC activation groups using a median split. Low
L-DLPFC patients ( $N=19,9$ real; 10 sham) showed no difference between sham and real stimulation for either response $\left(X^{2}=\right.$ $0.540, p=0.463)$ or remission $\left(X^{2}=0.006, p=0.937\right)$. However, high-L-DLPFC activation patients ( $\mathrm{N}=20,11$ real; 9 sham) were significantly more likely to respond to tDCS than sham stimulation $\left(X^{2}=5.69, p=0.017, \varphi=0.382\right.$; remission: $X^{2}=2.78, p=0.095$, $\varphi=0.267)$. A receiver operator characteristic analysis of the accuracy of predicting clinical response using baseline L-DLPFC activation revealed that the area-under-curve (AUC) for real stimulation was 0.856 (good) while for the sham group AUC = 0.417 (no better than chance).

Reliability

Activation in the L-DLPFC ROI (3-back > 1-back contrast) showed very good reliability between pre- and post-trial scans (combined $\mathrm{ICC}=0.67,95 \% \mathrm{Cl}=0.33-0.84, p=0.001)$. This was the case for both active (ICC $=0.69,95 \% \mathrm{Cl}=0.19-0.88)$ and sham $(\mathrm{ICC}=0.65$, $95 \% \mathrm{Cl}=-0.08-0.89)$ groups. However, activation in the right amygdala $\mathrm{ROI}$ (fearful > neutral contrast) showed poor reliability between scans (combined ICC $=-1.630,95 \% \mathrm{Cl}=$ -4.325 to $-0.299, p=0.996$; active: $\mathrm{ICC}=-1.83,95 \% \mathrm{Cl}=-6.35$ to -0.09 ; sham: ICC $=-1.26,95 \% \mathrm{Cl}=-6.04$ to -0.27 ) (Supplemental Fig. 2).

\section{DISCUSSION}

We conducted a mechanistic RCT of prefrontal tDCS combined with CBT for depression. We hypothesised that tDCS would enhance the therapeutic effect of CBT, compared with sham stimulation. Our results did not provide clear evidence for this; this may in part be due to the small sample size. On our mechanistic outcomes, we found that tDCS + CBT increased DLPFC activation relative to sham only during emotion processing, but not during working memory. Finally, we found a strong and specific association between pre-treatment L-DLPFC activation and subsequent clinical response, shedding light on the variability in our tDCS findings, and suggesting that it may be possible to identify which patients are most likely to respond to tDCS + CBT.

We did not find clear evidence that tDCS enhances the efficacy of CBT. While it is possible that our trial was underpowered (we identified moderately-sized odds ratios between active and sham groups (response: 2.16; remission: 3.65)), we found no effect of tDCS on self-reported depressive symptoms. Thus, the clinical relevance of tDCS combined with CBT remains to be determined in future, larger studies. It is also possible that the intervention itself is not clinically efficacious on average because of interindividual variability in response to tDCS. Indeed, a recent largescale international trial of tDCS for depression reported a null result (though that trial used a more typical daily or near-daily administration schedule, and a higher dosage) [17]. Therefore, one of the most important aspects of our trial was its mechanistic measures, which we could use to begin to explore such variability.

This trial was unusual in its inclusion of fMRI measurements. This design is central to understanding the possible mechanisms of $\mathrm{tDCS}+\mathrm{CBT}$, and can also illuminate sources of inter-individual 

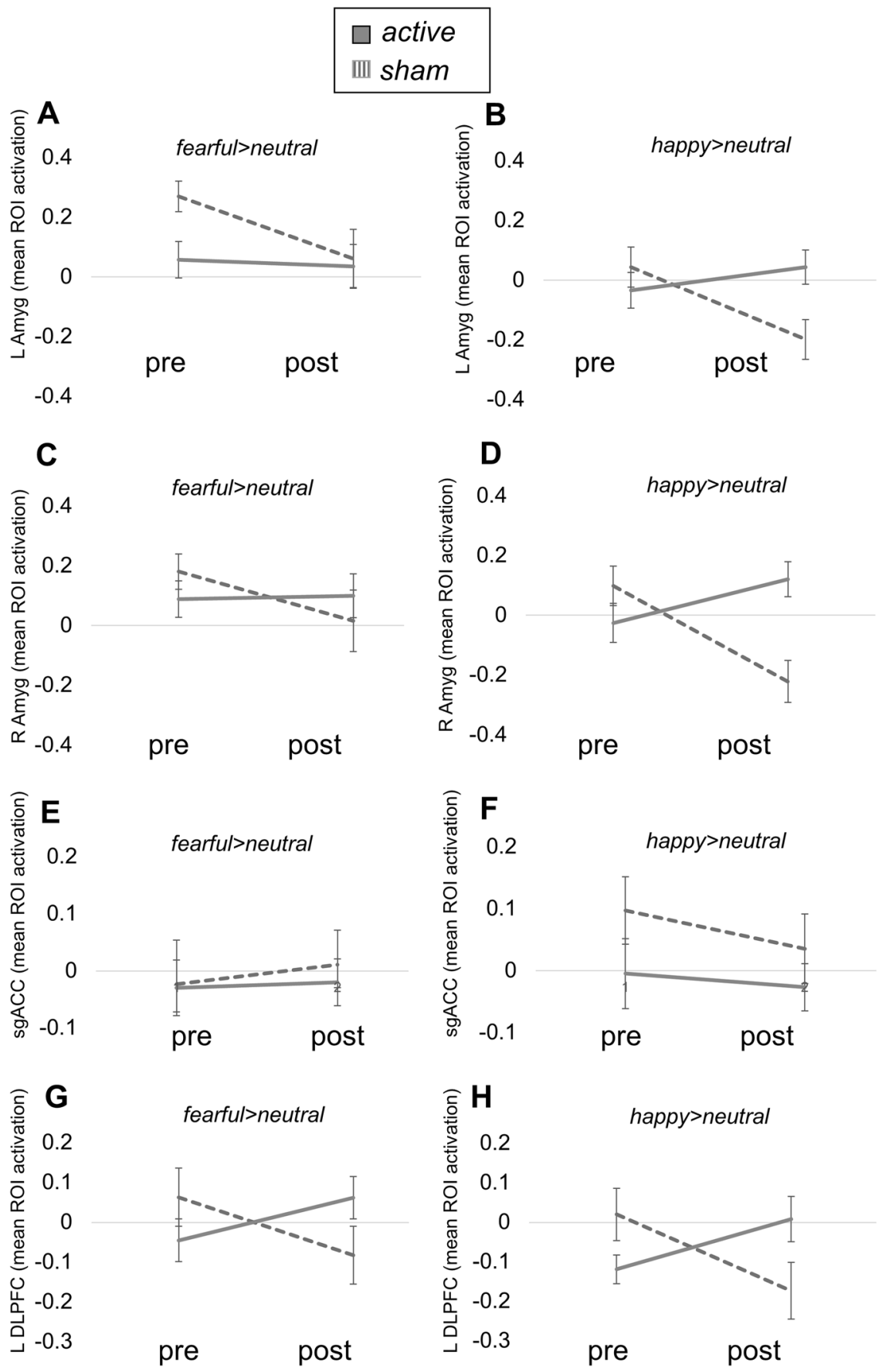

Fig. 3 Effect of intervention on neural activation during emotion processing. Activation in a priori regions of interest pre- and postintervention (orange solid lines = active tDCS; green dotted lines = sham tDCS) for the fearful vs neutral (left column) and happy vs neutral (right column) contrasts: left amygdala ( $\mathrm{Amyg}, \mathbf{a}, \mathbf{b})$; right amygdala (R Amyg, $\mathbf{C}$, d); subgenual anterior cingulate cortex (sgACC, e, f); and left dorsolateral prefrontal cortex ( $\mathrm{L}$ DLPFC, $\mathbf{g}, \mathbf{h})$. In the amygdalae (a-d) there was a significant time-by-stimulation condition interaction, with activation decreased from pre- to post-intervention in the sham condition only, with a similar pattern of results for fearful and happy stimuli. No significant effects were detected in the $\operatorname{sgACC}(\mathbf{e}, \mathbf{f})$. In the left DLPFC $(\mathbf{g}, \mathbf{h})$, there was a similar time-by-stimulation condition interaction to that in the amygdala

variability. Here, DLPFC activation during working memory increased substantially over the trial (irrespective of stimulation condition), consistent with a smaller study [39], to a level comparable with healthy volunteers. Additionally, specifically under active tDCS, right PPC activation increased from pre- to post-intervention. This might reflect long-range effects of tDCS on the parietal cortex [9], which is robustly activated by the n-back task [40]. Similarly, L-DLPFC and bilateral amygdala activation during emotion processing generally decreased over time under sham stimulation, but increased under active stimulation. Thus, although DLPFC activation during working memory did not increase more during active than during sham stimulation, we did observe treatment-dependent effects on the DLPFC during emotion processing.

Most importantly, we found that greater L-DLPFC activation prior to treatment was associated with greater subsequent 

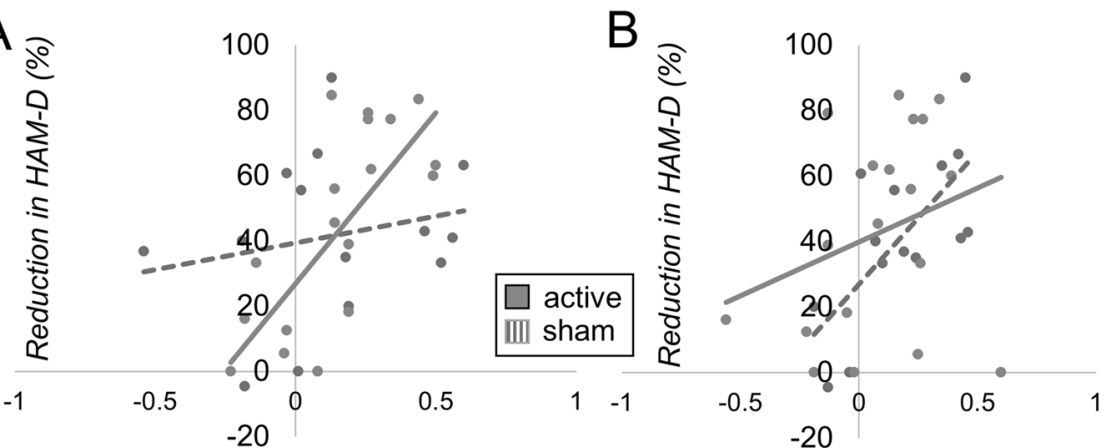

Left DLPFC activation (ROI)

Right amygdala activation (ROI)
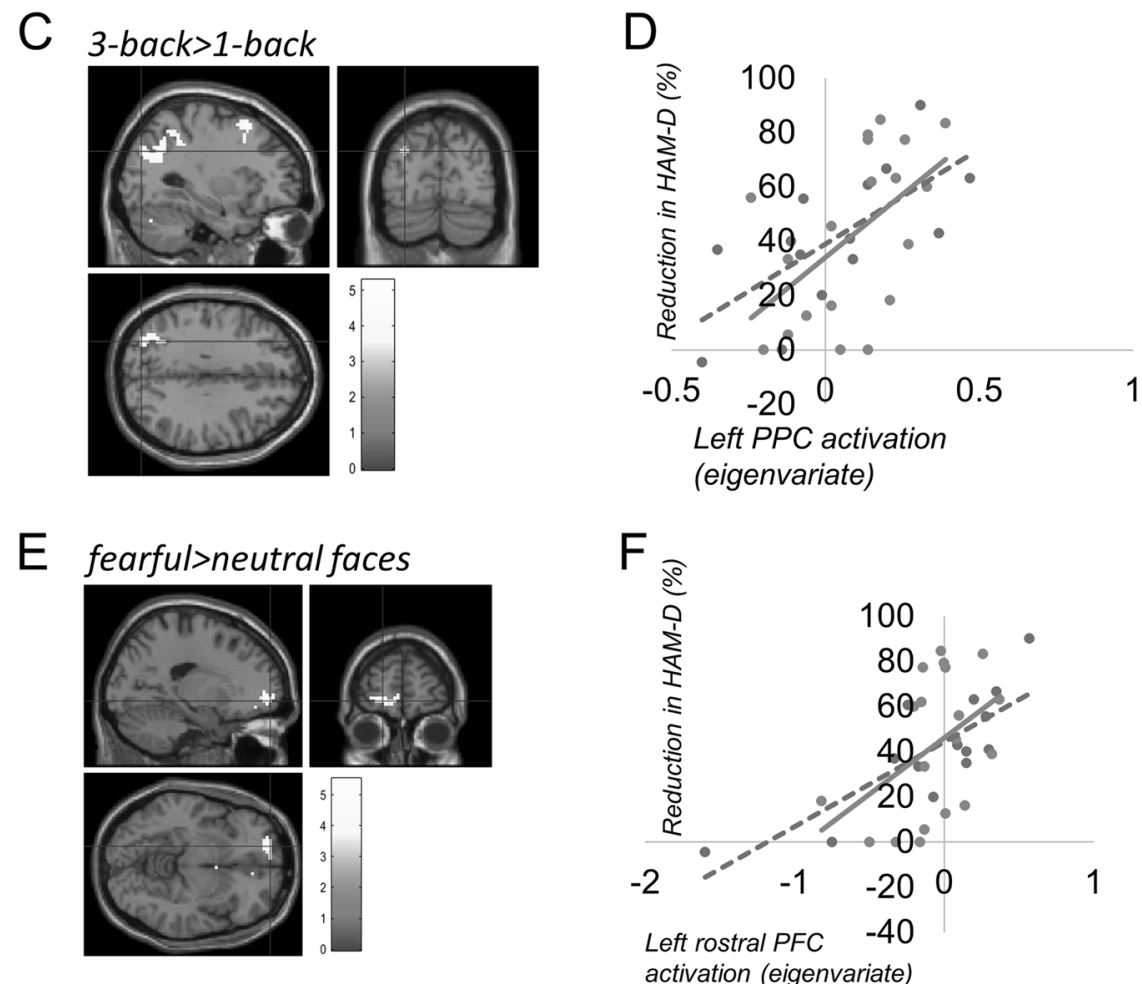

Fig. 4 ROI analysis: imaging biomarkers associated with clinical response. a Clinical response (percentage reduction in HAM-D) was associated with greater pre-treatment left DLPFC activation during working memory (3-back $>1$-back contrast) specifically in the active group $(r=0.711$, $p=0.001)$ but not the sham group $(r=0.205, p=0.482)$; interaction $(F(1,29)=6.83, p=0.014)$. b Clinical response was associated with greater pre-treatment right amygdala activation during emotion processing (fearful > neutral faces contrast) across both groups $(F(1,29)=7.69, p=$ $0.010)$. c Results of whole-brain exploratory analysis for regions predictive of outcome in the $n$-back task (3-back $>1$-back contrast) $(p=0.027$, cluster-corrected; note this does not fall below our stringent significance threshold of $p=0.025$ ). Crosshairs located at peak voxel in left posterior parietal cortex (L PPC). d For illustrative purposes only, scatterplot of relationship between L PPC activation (eigenvariate of cluster) and percent reduction in Hamilton Depression Rating Scale (HAM-D) over the trial in active (orange solid line) and sham (teal dotted line) groups. e Results of whole-brain exploratory analysis for regions predictive of outcome in the emotion processing task (fearful $>$ neutral contrast) ( $p=0.001$, cluster-corrected). Crosshairs located at peak voxel in left rostral prefrontal cortex (PFC); activation extends into bilateral OFC. f For illustrative purposes only, scatterplot of relationship between left rostral PFC activation (eigenvariate of cluster) and percent reduction in Hamilton Depression Rating Scale (HAM-D) over the trial in active and sham groups. For 5D and F, note that responses were extracted from clusters identified through a voxelwise analysis and therefore the magnitude of the correlation will be inflated (and thus is shown for illustrative purposes only)

symptomatic improvement, consistent with results from a small rTMS study [30]. Critically, this was only the case in patients receiving active stimulation, and this measure showed good reliability, a marker of potential utility as an fMRI "biomarker" [41]. Additionally, pre-treatment responses in the PPC (during working memory) and rostral PFC and right amygdala (during emotion processing) were associated with subsequent response to CBT, irrespective of stimulation condition (although note that the PPC result marginally missed statistical significance at our stringent FWE-corrected level of $p=0.025$ ). The amygdala result replicates the results of two previous neuroimaging investigations of CBT response [39, 42], although puzzlingly the right amygdala also shows extremely poor test-retest reliability [41].

Our results suggest the L-DLPFC may have potential in predicting response to neurostimulation in depression, a result confirmed by significant effects on our primary outcome (HAM-D response) for the high-L-DLPFC group only, and by high accuracy of an ROC analysis using baseline L-DLPFC activation. In contrast, n-back working memory performance was not associated with improved clinical response. The high within-subject reliability 
of L-DLPFC activation fulfils a vital criterion for a clinically useful predictor, and suggests the L-DLPFC blood-oxygen-level dependent (BOLD) response is more stable than other regions proposed as 'biomarkers', such as the sgACC and amygdala, which appear to have poor within-subject reliability [41], a finding we replicated here.

Individual differences in baseline DLPFC activation may contribute to the notable inconsistencies in the broader field of prefrontal tDCS [23]. Speculatively, while clinical response to our low dose of tDCS $(1 \mathrm{~mA})$ is associated with high-L-DLPFC activation at baseline, other dosages (for instance, the $2.5 \mathrm{~mA}$ used in a recent negative international clinical trial [17]) might show a different, or even reversed, relationship with DLPFC activation, making incorporation of mechanistic measures in future trials essential.

\section{Limitations and future directions}

Despite the strengths of our mechanistic design, there are important limitations, including relatively weak current strength and a fronto-extracephalic electrode montage. This montage results in comparatively diffuse frontal stimulation according to current flow models [33]. We chose this montage because in a preliminary open-label study, it was substantially more effective for depression than a bifrontal tDCS montage [34]. We have previously shown an identical montage and current strength to have cognitive effects in healthy controls [43]. Recent work has confirmed the physiological validity of unilateral $1 \mathrm{~mA}$ montages, failing to show physiological validity for bilateral stimulation or 2 $\mathrm{mA}$ intensity conditions [35], despite their efficacy in previous depression trials $[12,44]$. It also bears mentioning that we found increased redness and itching observed in the real versus sham conditions; however, patients were worse than chance at guessing their stimulation condition (other side effects were significantly more common in the sham condition).

Our weekly stimulation schedule could also have contributed to our null clinical result [11-14] (weekly CBT sessions is typical for NHS outpatients with moderate-to-severe depression). Furthermore, there is inherent heterogeneity between CBT therapists (confirmed here, despite efforts to standardise treatment); although including therapist as a covariate did not change our analyses.

Finally, we discovered a potential predictor of treatment outcome (baseline L-DLPFC activation), but it is as-yet unclear how this potential predictor relates to cognitive factors; importantly, baseline working memory was not predictive of treatment outcome. It is possible that DLPFC activation during the n-back task is reflective of a more general cold cognitive capacity than working memory ability per se; other cognitive measures of executive function (or perhaps a combined measure) may be better predictors of outcome than the $n$-back. One of the most important factors to establish, if tDCS is an effective intervention for depression, is the particular neural target it engages. While the most intuitive candidate is the region stimulated, our result failed to show any differential treatment effects on this region. Instead, we found a general increase in bilateral DLPFC activation regardless of tDCS condition. Future work should explore the contribution of baseline variables to this change in DLPFC activation. However, it is certainly possible that while the immediate neural effects of tDCS occur within the stimulated region (as previous work would suggest [9]), the longer-term mechanisms driving its clinical effect may be more distally located (for example, in the parietal cortex, as suggested by our exploratory whole-brain analysis).

\section{CONCLUSION}

Our data do not provide clear support for the use of tDCS to augment CBT in depression in all patients, but we discovered a biomarker explaining variability in tDCS response: baseline activation in the area of stimulation (L-DLPFC). This biomarker appears relatively strong and specific to active stimulation. Our data speak to the potential utility of neuroscience techniques in developing novel mental health interventions; incorporating cognitive neuroscience measures into clinical trials could allow simultaneous assessment of clinical efficacy and detection of potential biomarkers of treatment response. Ultimately, these data could guide patient selection in larger clinical trials, and, if confirmed, inform clinical use of tDCS in depression.

\section{FUNDING AND DISCLOSURE}

This work was supported by the Brain and Behavior Research Foundation (grant number 20162) to JPR and a Brain Research Trust PhD studentship awarded to CLN, and supported by the National Institute for Health Research University College London Hospitals Biomedical Research Centre (SP). JPR consults for Cambridge Cognition, Takeda Ltd and GE. The authors declare no competing interests.

\section{DATA AVAILABILITY}

All data discussed in the paper will be made available to any reader who enters into a managed access arrangement. Ethical and data governance restrictions prevent the data from being shared in a public repository (all data are patient data collected under UK National Health Service ethics and patients did not give consent for open sharing). All data will be shared freely following a formal Data Sharing Agreement with University College London. Researchers who wish to access the data should contact the senior author (j.roiser@ucl.ac.uk).

\section{ACKNOWLEDGEMENTS}

The authors would like to thank research assistants Jessica Alylward and Alan Gray for their help with data collection. They are also extremely grateful to the NHS clinicians Michaela Thomas, Dr Chinea Eziefula, Carlo Gittens, Laura Franklin, Dr Karen Katz, Dr Robin Weedon, Dr Jeremy Handel, Dr Flavia Cigolla, Akbar Jamil, Dr Isabella Foustanos, Dr Abi Harris, Dr Sukhvinder Virdee, Dr Jessica Wilner-Reid, and Dr Joshua Buckman, as well as all the patients who generously volunteered their time for the trial.

\section{AUTHOR CONTRIBUTIONS}

CLN, JPR, NL, SP, VV, and CC designed the study, CLN, CH, TL, and JL collected data, CLN analysed data, CLN and JPR wrote the paper with contributions from all the other authors.

\section{ADDITIONAL INFORMATION}

Supplementary Information accompanies this paper at (https://doi.org/10.1038/ s41386-019-0401-0).

Publisher's note: Springer Nature remains neutral with regard to jurisdictional claims in published maps and institutional affiliations.

\section{REFERENCES}

1. Kendall PC, Hollon SD. Cognitive-behavioral interventions: theory, research, and procedures. vol. 21. New York, NY: Academic Press; 2013.

2. DeRubeis RJ, Hollon SD, Amsterdam JD, Shelton RC, Young PR, Salomon RM, et al Cognitive therapy vs medications in the treatment of moderate to severe depression. Arch Gen Psychiatry. 2005;62:409-16.

3. Frye MA, Grunze H, Suppes T, McElroy SL, Keck PE Jr, Walden J, et al. A placebocontrolled evaluation of adjunctive modafinil in the treatment of bipolar depression. Am J Psychiatry. 2007;164:1242-9.

4. Vedeniapin A, Cheng L, George MS. Feasibility of simultaneous cognitive behavioral therapy (CBT) and left prefrontal rTMS for treatment resistant depression. Brain Stimul. 2010;3:207.

5. D'Urso G, Mantovani A, Micillo M, Priori A, Muscettola G. Transcranial direct current stimulation and cognitive-behavioral therapy: evidence of a synergistic effect in treatment-resistant depression. Brain Stimul. 2013;6:465-7. 
6. Wang $X-L$, Du $M-Y$, Chen T-L, Chen Z-Q, Huang X-Q, Luo $Y$, et al. Neural correlates during working memory processing in major depressive disorder. Prog NeuroPsychopharmacol Biol Psychiatry. 2015;56:101-8.

7. Hooley JM, Gruber SA, Scott LA, Hiller JB, Yurgelun-Todd DA. Activation in dorsolateral prefrontal cortex in response to maternal criticism and praise in recovered depressed and healthy control participants. Biol Psychiatry. 2005;57:809-12.

8. Kerestes R, Ladouceur $C$, Meda $S$, Nathan $\mathrm{P}$, Blumberg $\mathrm{H}$, Maloney $\mathrm{K}$, et al. Abnormal prefrontal activity subserving attentional control of emotion in remitted depressed patients during a working memory task with emotional distracters. Psychol Med. 2012;42:29-40.

9. Stagg CJ, Lin RL, Mezue M, Segerdahl A, Kong Y, Xie J, et al. Widespread modulation of cerebral perfusion induced during and after transcranial direct current stimulation applied to the left dorsolateral prefrontal cortex. J Neurosci. 2013:33:11425-31.

10. Nord CL, Lally N, Charpentier CJ. Harnessing electric potential: DLPFC tDCS induces widespread brain perfusion changes. Front Syst Neurosci. 2013;7:99.

11. Boggio PS, Rigonatti SP, Ribeiro RB, Myczkowski ML, Nitsche MA, Pascual-Leone $A$, et al. A randomized, double-blind clinical trial on the efficacy of cortical direct current stimulation for the treatment of major depression. Int J Neuropsychopharmacol. 2008;11:249-54.

12. Brunoni A, Valiengo L, Baccaro A, Zanao TA, de Oliveira JF, Goulart A, et al. The sertraline vs electrical current therapy for treating depression clinical study: results from a factorial, randomized, controlled trial. JAMA Psychiatry. 2013;70:383-91.

13. Fregni F, Boggio PS, Nitsche MA, Marcolin MA, Rigonatti SP, Pascual-Leone A. Treatment of major depression with transcranial direct current stimulation. Bipolar Disord. 2006;8:203-4.

14. Loo CK, Alonzo A, Martin D, Mitchell PB, Galvez V, Sachdev P. Transcranial direct current stimulation for depression: 3-week, randomised, sham-controlled trial. $\mathrm{Br}$ J Psychiatry. 2012;200:52-9.

15. Nord CL, Roiser J. Non-invasive direct current brain stimulation: the evidence behind the hype. Adv Clin Neurosci Rehabil. 2015;15:9-11.

16. Shiozawa P, Fregni F, Benseñor IM, Lotufo PA, Berlim MT, Daskalakis JZ, et al. Transcranial direct current stimulation for major depression: an updated systematic review and meta-analysis. Int. J. Neuropsychopharmacol. 2014; 17:1443-52.

17. Loo CK, Husain MM, McDonald WM, Aaronson S, O'Reardon JP, Alonzo A, et al. International randomized-controlled trial of transcranial Direct Current Stimulation in depression. Brain Stimul. 2018;11:125-33.

18. Dockery CA, Hueckel-Weng R, Birbaumer N, Plewnia C. Enhancement of planning ability by transcranial direct current stimulation. J Neurosci. 2009;29:7271-7.

19. Metuki N, Sela T, Lavidor M. Enhancing cognitive control components of insight problems solving by anodal tDCS of the left dorsolateral prefrontal cortex. Brain Stimul. 2012;5:110-5.

20. Fregni $F$, Boggio PS, Nitsche $M$, Bermpohl F, Antal A, Feredoes $E$, et al. Anodal transcranial direct current stimulation of prefrontal cortex enhances working memory. Exp Brain Res. 2005;166:23-30.

21. Lally N, Nord CL, Walsh V, Roiser JP. Does excitatory fronto-extracerebral tDCS lead to improved working memory performance? F1000Res. 2013;2:219.

22. Oliveira JF, Zanao TA, Valiengo L, Lotufo PA, Bensenor IM, Fregni F, et al. Acute working memory improvement after tDCS in antidepressant-free patients with major depressive disorder. Neurosci Lett. 2013;537:60-64.

23. Horvath JC, Forte JD, Carter O. Quantitative review finds no evidence of cognitive effects in healthy populations from single-session transcranial direct current stimulation (tDCS). Brain Stimul. 2015;8:535-50.
24. Tremblay S, Lepage J-F, Latulipe-Loiselle A, Fregni F, Pascual-Leone A, Théoret $\mathrm{H}$. The uncertain outcome of prefrontal tDCS. Brain Stimul. 2014;7:773-83.

25. Price AR, Hamilton RH. A Re-evaluation of the cognitive effects from singlesession transcranial direct current stimulation. Brain Stimul. 2015;8:663-5.

26. Segrave R, Arnold S, Hoy K, Fitzgerald P. Concurrent cognitive control training augments the antidepressant efficacy of tDCS: a pilot study. Brain Stimul. 2014;7:325-31.

27. Welch ES, Weigand A, Hooker JE, Philip NS, Tyrka AR, Press DZ, et al. Feasibility of computerized cognitive-behavioral therapy combined with bifrontal transcranial direct current stimulation for treatment of major depression. Neuromodulation. 2018. https://onlinelibrary.wiley.com/doi/full/10.1111/ner.12807.

28. Dunlop BW, Mayberg HS. Neuroimaging-based biomarkers for treatment selection in major depressive disorder. Dialog Clin Neurosci. 2014;16:479.

29. Roiser JP, Elliott R, Sahakian BJ. Cognitive mechanisms of treatment in depression. Neuropsychopharmacology. 2012;37:117-36.

30. Weiduschat N, Dubin MJ. Prefrontal cortical blood flow predicts response of depression to rTMS. J Affect Disord. 2013;150:699-702.

31. Nitsche MA, Paulus W. Sustained excitability elevations induced by transcranial DC motor cortex stimulation in humans. Neurology. 2001;57:1899-901.

32. O'Nions EJ, Dolan RJ, Roiser JP. Serotonin transporter genotype modulates subgenual response to fearful faces using an incidental task. J Cogn Neurosci. 2011;23:3681-93.

33. Laakso I, Tanaka S, Mikkonen M, Koyama S, Sadato N, Hirata A. Electric fields of motor and frontal tDCS in a standard brain space: a computer simulation study. Neuroimage. 2016;137:140-51.

34. Martin DM, Alonzo A, Mitchell PB, Sachdev P, Gálvez V, Loo CK. Frontoextracephalic transcranial direct current stimulation as a treatment for major depression: an open-label pilot study. J Affect Disord. 2011;134:459-63.

35. Parkin BL, Bhandari M, Glen JC, Walsh V. The physiological effects of Transcranial Electrical Stimulation do not apply to parameters commonly used in studies of Cognitive Neuromodulation. Neuropsychologia. 2018. https://www.sciencedirect. com/science/article/pii/S0028393218301234.

36. Ambrus GG, Al-Moyed H, Chaieb L, Sarp L, Antal A, Paulus W. The fade-in-short stimulation-fade out approach to sham tDCS-reliable at $1 \mathrm{~mA}$ for naive and experienced subjects, but not investigators. Brain Stimul. 2012;5:499-504.

37. Hollon SD, Stewart MO, Strunk D. Enduring effects for cognitive behavior therapy in the treatment of depression and anxiety. Annu Rev Psychol. 2006;57:285-315.

38. Brunoni AR, Amadera J, Berbel B, Volz MS, Rizzerio BG, Fregni F. A systematic review on reporting and assessment of adverse effects associated with transcranial direct current stimulation. Int J Neuropsychopharmacol. 2011;14:1133-45.

39. Siegle GJ, Thompson W, Carter CS, Steinhauer SR, Thase ME. Increased amygdala and decreased dorsolateral prefrontal BOLD responses in unipolar depression: related and independent features. Biol Psychiatry. 2007;61:198-209.

40. Owen AM, McMillan KM, Laird AR, Bullmore E. N-back working memory paradigm: a meta-analysis of normative functional neuroimaging studies. Hum Brain Mapp. 2005;25:46-59.

41. Nord CL, Gray A, Charpentier C, Robinson O, Roiser J Unreliability of putative fMRI biomarkers during emotional face processing. Neuroimage. 2017;156:119-27.

42. Siegle GJ, Carter CS, Thase ME. Use of FMRI to predict recovery from unipolar depression with cognitive behavior therapy. Am J Psychiatry. 2006:163:735-38.

43. Nord CL, Forster S, Halahakoon DC, Penton-Voak IS, Munafò MR, Roiser JP. Prefrontal cortex stimulation does not affect emotional bias, but may slow emotion identification. Soc Cogn Affect Neurosci. 2017;12:839-47.

44. Brunoni AR, Moffa AH, Sampaoi-Junior B, Borrione L, Moreno ML, Fernandes RA, et al. Trial of electrical direct-current therapy versus escitalopram for depression. N Engl J Med. 2017;376:2523-33. 